\title{
The spectrum of the independent Metropolis-Hastings algorithm
}

\author{
Jørund Gåsemyr
}

Revised January 2004

\begin{abstract}
In this paper we perform a spectral analysis for the kernel operator associated with the transition kernel for the Metropolis-Hastings algorithm that uses a fixed, location independent proposal distribution. Our main result is to establish the spectrum of the kernel operator $T$ in the continuous case. In the case of finite state spaces we give the eigenvalues and eigenvectors, thereby filling in a few missing details in the slightly incomplete analysis in Liu (1996). The spectrum of $T$ is of interest for several reasons. Performance measures such as total variation distance after a finite number of iterations, rate of convergence in total variation norm, acceptance rate, autocovariances and efficiency can be expressed in terms of the spectrum of $T$.
\end{abstract}

Key words: Independent chain, Markov Chain Monte Carlo, Metropolized independent sampling.

\section{Introduction}

Let $\pi$ be a probability density with respect to a $\sigma$ - finite measure $\mu$ on a state space $\Omega$. Let $q$ be another density, acting as a proposal distribution for a Metropolis-Hastings algorithm (Metropolis et al (1953), Hastings (1970)). This algorithm produces a Markov chain $\left(X_{t}\right), t=1,2, \ldots$, with $\pi$ as stationary distribution by drawing a candidate $y$ from $q$ for the successor $X_{t+1}$ of $X_{t}=x$, then moving to $y$ with probability $\alpha(x, y)=$ $\min \{1, \pi(y) q(x) /(\pi(x) q(y))\}$, and remaining in $x$ with probability $1-\alpha(x, y)$. 
The resulting Markov chain was called an independent chain (IC) in Tierney (1994). The procedure was referred to as Metropolized independent sampling in Liu (1996), and as independent Metropolis-Hastings (IMH) in Holden et al. (2002)).

We start by introducing some key concepts and notation. For $x \in \Omega$ define the importance weight $w(x)=\pi(x) / q(x)$. Let $B_{w}=\{x: w(x) \leq w\}$, $B_{w^{-}}=\{x: w(x)<w\}, 0 \leq w<\infty$. Furthermore, define

$$
\lambda_{w}=\int_{B_{w}}(q(y)-\pi(y) / w) d \mu(y)=\int_{B_{w^{-}}}(q(y)-\pi(y) / w) d \mu(y)
$$

The interpretation of $\lambda_{w}$ is that it is the probability of remaining at the current state $x$, given that $w(x)=w$, i.e. $P\left(X_{t+1}=x \mid X_{t}=x, w(x)=w\right)$. The transition kernel for the Markov chain associated with the IMH algorithm is given by

$$
\begin{aligned}
K(x, A)= & \int_{A} q(y) d \mu(y) \\
& +\int_{A \cap B_{w(x)}}(-q(y)+\pi(y) / w(x)) d \mu(y)+\lambda_{w(x)} I(x \in A)
\end{aligned}
$$

The kernel operator on $L^{2}(\pi)$ associated with this transition kernel is defined by

$$
\begin{aligned}
T f(x)= & \int_{\Omega} f(y) k(x, d y)=\int_{\Omega} f(y) q(y) d \mu(y) \\
& +\int_{B_{w(x)}} f(y)(-q(y)+\pi(y) / w(x)) d \mu(y)+\lambda_{w(x)} f(x)
\end{aligned}
$$

Sometimes it is more convenient to write this in the form

$$
\begin{aligned}
T f(x)= & \int_{B_{w(x)}^{c}} f(y) q(y) d \mu(y) \\
& +\int_{B_{w(x)}}(f(y) \pi(y) / w(x)) d \mu(y)+\lambda_{w(x)} f(x)
\end{aligned}
$$

The eigenvalues of $T$ are of interest for several reasons. It is shown in Liu (1996) and Hauge (2002) that performance measures such as total variation distance after a finite number of iterations, rate of convergence in total variation norm, acceptance rate, autocovariances and efficiency can be expressed 
in terms of the eigenvalues in the discrete case, and these results are generalized to the continuous case in Gåsemyr (2004), which is a natural companion to the present paper. The eigenvalues and eigenvectors of $T$ were studied in Liu (1996) in the case when the state space $\Omega$ is finite and the ordering on $\Omega$ defined by $w(x)$ is strict. Liu showed that the eigenvalues are the non zero values of $\lambda_{w(x)}, x \in \Omega$, as well as the trivial eigenvalue 1 . He also found all the eigenvectors in this case. In section 2 of the present paper we generalize this to the case when $w(x)$ is not necessarily monotonic, and in section 4 we characterize the spectrum in the general case of an arbitrary state space with an arbitrary proposal distribution $q$ with the same support as $\pi$. We will assume without loss of generality that both $\pi$ and $q$ are positive on all of $\Omega$.

A central object in Liu's analysis for finite spaces $\Omega$ is the set $\{w(x): x \in$ $\Omega$ \} of importance weights. The appropriate extension to the general case is the set

$$
\mathcal{W}=\left\{w: \pi\left(B_{w+1 / n}-B_{w-1 / n}\right)>0 \text { for all } n>0\right\}
$$

Then $\mathcal{W}$ is a closed set which can be viewed as the support of a real probability measure defined by the cumulative distribution function $F_{\pi}(w)=\pi\left(B_{w}\right)$. Obviously, $\pi(\{x: w(x) \in \mathcal{W}\})=1$. Redefining $q$ on a set of $\pi$-measure 0 causes no essential changes, and we will assume without loss of generality that $w(x) \in \mathcal{W}$ for each $x \in \Omega$. Equivalently, we assume $w^{-1}(\mathcal{W})=\Omega$. The set $\mathcal{W}$ is bounded by the numbers $w_{*}=\inf (\mathcal{W}), w^{*}=\sup (\mathcal{W})$. The possibility $w^{*}=\infty$ is not excluded. Define

$$
\Lambda=\left\{\lambda_{w}: w \in \mathcal{W}\right\}
$$

We then have $\lambda_{w_{*}}=0, \lambda_{w^{*}}=1-1 / w^{*}$ if $w^{*}<\infty$, and $\Lambda \subseteq\left[0,1-1 / w^{*}\right]$. The mapping $w \rightarrow \lambda_{w}$ on $\mathcal{W}$ is continuous, and therefore the image $\Lambda$ of this mapping is closed if $\mathcal{W}$ is bounded. If $w^{*}=\infty$, we have that $\Lambda \cap[0, a]$ is a closed set for every $a<1$.

It is conjectured in Liu (1996) that in the continuous case the spectrum of $T$, i.e. the set of $\lambda$ such that $T-\lambda I$ is not invertible, is the whole interval $\left[0,1-1 / w^{*}\right]$, except for the trivial value 1 . In this paper we show that the spectrum of $T$ is actually $\Lambda \cup\{1\}$. The set $\Lambda$ may be disconnected, and hence Liu's conjecture is disproved. 


\section{The case of finite state spaces}

The case of finite $\Omega$, implying finite $\mathcal{W}$, was analysed in Liu (1996). In this section we fill in a few missing details. In principle, we may have $\mathcal{W}$ finite also in the continuous case. In fact, an analysis of this eventuality turns out to be needed as a tool in the examination of the general continuous case. We therefore start out by only assuming that $\mathcal{W}$ is finite, making no assumptions on $\Omega$. Some further results for finite $\mathcal{W}$, as well as a special case of countable $\mathcal{W}$, are developed in the next section.

Suppose $w_{*}=w_{1}<w_{2}<\ldots<w_{r}=w^{*}$. Then also $0=\lambda_{w_{1}}<\lambda_{w_{2}}<$ $\ldots<\lambda_{w_{r}}<1$. Define

$$
C_{k}=\left\{x: w(x)=w_{k}\right\} \text {, i.e. } C_{1}=B_{w_{1}}, C_{k}=B_{w_{k}}-B_{w_{k-1}}, k=2,3, \ldots, r
$$

Then $q(y)=\pi(y) / w_{k}$ for $y \in C_{k}$, and hence for any $f \in L^{2}(\pi)$ and $x \in C_{k}$

$$
\begin{aligned}
\int_{B_{w_{k}}}(q(y) & \left.-\pi(y) / w_{k}\right) f(y) d \mu(y)=\int_{B_{w(x)}}\left(q(y)-\pi(y) / w_{k}\right) f(y) d \mu(y) \\
= & \int_{B_{w_{k-1}}}\left(q(y)-\pi(y) / w_{k}\right) f(y) d \mu(y)
\end{aligned}
$$

Lemma 1 If $\lambda$ is an eigenvalue for $t$, then $\lambda \in \Lambda$, or $\lambda=1$.

Proof: Let $\lambda$ be an eigenvalue with non trivial eigenvector $f$. Consider $x \in C_{1}$. Then $\lambda_{w(x)}=\lambda_{w_{1}}=0$ and $q(y)=\pi(y) / w(x)$ on $B_{w(x)}$. Hence, it follows from (3) that $\lambda f(x)=T f(x)=\int_{\Omega} f(y) q(y) d \mu(y)$. This implies that either is $f$ constant on $C_{1}$, or $\int_{\Omega} f(y) q(y) d \mu(y)=\lambda=0=\lambda_{w_{1}} \in \Lambda$. If $f$ is constant, we have to consider two different cases:

Case 1: $f(x)$ is non zero on $C_{1}$. Assume without loss of generality that $f(x)=1$ for $x \in C_{1}$. Then $\lambda=\int_{\Omega} f(y) q(y) d \mu(y)$. Consider now instead $x \in C_{2}$. Then $w(x)=w_{2}$, and by (3), (8) and (1)

$$
\lambda f(x)=T f(x)=\lambda-\lambda_{w_{2}}+\lambda_{w_{2}} f(x),
$$

so that $\left(\lambda-\lambda_{w_{2}}\right) f(x)=\lambda-\lambda_{w_{2}}$. This implies that either $\lambda=\lambda_{w_{2}} \in \Lambda$, or $f$ is constant and equal to 1 also on $C_{2}$, i.e. on all of $B_{w_{2}}$. Continuing inductively, considering in turn $x \in C_{3}, C_{4}, \ldots, C_{r}$, we conclude that either we have $\lambda=\lambda_{w_{k}}$ for some $k \in\{1, \ldots, r\}$, or $f$ is identically 1 on $\Omega$, in which case $\lambda=1$. 
Case 2: $f(x)=0$ for $x \in C_{1}$. Then $\int_{\Omega} f(y) q(y) d \mu(y)=0$. Considering $x \in C_{2}$ it follows that $\lambda f(x)=\lambda_{w_{2}} f(x)$. This means that either $\lambda=\lambda_{w_{2}}$, or $f$ is 0 also on $c_{2}$. Proceeding inductively as in case 1 , we conclude that $\lambda \in \Lambda$, completing the proof.

The proof of lemma 1 suggests candidates for eigenvectors for $T$. In fact, the different possibilities considered in case 1 are consistent with the eigenvectors given in lemma 2 below, whereas the possibilities considered in case 2 are consistent with eigenvectors if the corresponding eigenspaces are not one dimensional.

Lemma 2 Define $B_{w_{0}}=\emptyset$, and suppose $f \in L^{2}(\pi)$ satisfies

(i) $f(x)=1$ for $x \in B_{w_{k-1}}$

(ii) $\int_{C_{k}} f(y) \pi(y) d \mu(y)=-\pi\left(B_{w_{k-1}}\right)$

(iii) $f(x)=0$ for $x \in B_{w_{k}}^{c}$

for some $k \in\{1, \ldots, r\}$. Then $f$ is an eigenvector for $T$ with eigenvalue $\lambda_{w_{k}}$. In particular, if $\int_{C_{1}} f(y) \pi(y) d \mu(y)=0$ and $f(x)=0$ for $x \notin C_{1}=B_{w_{1}}$, then $f$ is in the kernel of $T$, i.e. is an eigenvector with eigenvalue 0.

Proof: Suppose $f$ satisfies the condition with $k=1$. Then $\lambda_{w(x)} f(x)=0$ for all $x$, since $\lambda_{w(x)}=0$ if $x \in C_{1}$, and $f(x)=0$ if $x \in C_{1}^{c}$. Hence, for any $x$ (4) gives

$$
\begin{aligned}
T f(x) & =\int_{B_{w(x)}^{c}} q(y) f(y) d \mu(y)+\int_{B_{w(x)}}(\pi(y) / w(x)) f(y) d \mu(y)+\lambda_{w(x)} f(x) \\
& =0+\int_{B_{w_{1}}}(\pi(y) / w(x)) f(y) d \mu(y)+0=0
\end{aligned}
$$

proving the case $k=1$. Fix $k>1$. Note that from (7), (i) and (ii) we have

$$
\int_{B_{w_{k}}} f(y) \pi(y) d \mu(y)=\pi\left(B_{w_{k-1}}\right)+\int_{C_{k}} f(y) \pi(y) d \mu(y)=0
$$

From (iii), (8) and (9) it follows that

$$
\begin{gathered}
\int_{\Omega} q(y) f(y) d \mu(y)=\int_{B_{w_{k-1}}}\left(q(y)-\pi(y) / w_{k}\right) d \mu(y) \\
+\int_{B_{w_{k}}}\left(\pi(y) / w_{k}\right) f(y) d \mu(y)=\lambda_{w_{k}}
\end{gathered}
$$


Consider first $x \in B_{w_{k-1}}$. Then from (3)

$$
T f(x)=\lambda_{w_{k}}-\lambda_{w(x)}+\lambda_{w(x)} \cdot 1=\lambda_{w_{k}}=\lambda_{w_{k}} f(x) .
$$

Next, consider $x \in C_{k}$. Then from (3)

$$
T f(x)=\lambda_{w_{k}}-\lambda_{w_{k}}+\lambda_{w(x)} f(x)=\lambda_{w_{k}} f(x) .
$$

Finally, consider $x \in B_{w_{k}}^{c}$. Then

$$
\begin{gathered}
T f(x)=\int_{B_{w(x)}^{c}} q(y) f(y) d \mu(y)+\int_{B_{w(x)}} \pi(y) / w(x) f(y) d \mu(y)+\lambda_{w(x)} \cdot 0 \\
=\int_{B_{w_{k}}} \pi(y) / w(x) f(y) d \mu(y)=0=\lambda_{w_{k}} f(x)
\end{gathered}
$$

having used (9) again. This completes the proof.

Lemma 2 shows that $\lambda_{w_{2}}, \ldots, \lambda_{w_{r}}$ are eigenvalues for $T$. In addition, it follows from (ii) that $\lambda_{w_{1}}$ is an eigenvalue if there exists a non trivial $f$ such that $\int_{C_{1}} f(y) \pi(y) d \mu(y)=0$, which is the case unless $C_{1}$ has its entire probability mass concentrated in a single state. Furthermore, 1 is an eigenvalue for the constant function $\mathbf{1}$.

We can now deal easily with the case of finite state spaces. For any finite set $A$, denote by $|A|$ the number of elements of $A$.

Theorem 1 Suppose $\Omega$ is finite, with $|\Omega|=N$, and $\mathcal{W}=\left\{w_{1}, \ldots, w_{r}\right\}$, where $w_{1}<w_{2}<\cdots<w_{r}$. For $k=1, \ldots, r$, denote by $L_{k}$ the set of functions $f$ satisfying

(i) $f$ is constant on $B_{w_{k-1}}$

(ii) $\sum_{x \in C_{k}} f(x) \pi(x)=-\sum_{x \in B_{w_{k-1}}} f(x) \pi(x)$

(iii) $f(x)=0$ for $x \in B_{w_{k}}^{c}$

Then $L_{1}, L_{2}, \ldots, L_{r}, L_{r+1}=\mathbf{R} \cdot \mathbf{1}$ are orthogonal eigenspaces for $T$, spanning all of $L^{2}(\pi)=\mathbf{R}^{N}$, with eigenvalues $\lambda_{w_{1}}=0, \lambda_{w_{2}}, \ldots, \lambda_{w_{r}}, 1$ and dimensions $\left|C_{1}\right|-1,\left|C_{2}\right|, \ldots,\left|C_{r}\right|, 1$ respectively. 
Proof: It follows from lemma 2 that $L_{k}$ is an eigenspace for $T$ with eigenvalue $\lambda_{w_{k}}$ for each $k$. Clearly, the dimension of $L_{1}$ is $\left|C_{1}\right|-1$, whereas the dimension of $L_{k}$ is $\left|C_{k}\right|$ for $2 \leq k \leq r$. If $f \in L_{k}, g \in L_{k^{\prime}}, k^{\prime}>k$, then $\sum_{x \in \Omega} f(x) g(x) \pi(x)=\sum_{x \in B_{w_{k}}} f(x) \pi(x)=0$ by (9). Since $\left|C_{1}\right|-1+\left|C_{2}\right|+$ $\cdots+\left|C_{r}\right|+1=|\Omega|=N$, the proof is complete.

Note that if $w(x)$ defines a strict ordering on $\Omega$, so that $r=N$, then each eigenspace $L_{k}, k \geq 2$, has dimension 1 , and $\lambda_{w_{0}}=0$ is not an eigenvalue. This is in accordance with the results of Liu (1996).

\section{The case of a finite or countable set $\mathcal{W}$ of importance weights}

Throughout this section we will assume that $\mathcal{W}$ is either finite, or is countable, bounded and has no accumulation points other than $w_{*}$. In the latter case, $\mathcal{W}$ can be arranged in order correspondance with the ordered set $\{\ldots,-2,-1,0,-1, \ldots, N-1, N\}$. Here, $N$ is arbitrary and could be taken to be 0 , but we denote the maximal element by $N$ to keep the notational difference to the finite case as small as possible. For the following lemma we make the even stronger assumption that $\Omega$ itself is finite or countable and can be strictly ordered in the same way:

Lemma 3 Suppose $\Omega$ is either finite or countable, consisting of $\left\{x_{k}\right\}$, with $k$ ranging either from 1 to $N$ or from $-\infty$ to $N$. Suppose also that the sequence $\left(w_{k}=w\left(x_{k}\right)\right)$ is strictly increasing, hence necessarily bounded by $w_{N}=w^{*}$. Then

(i) the function $f_{k}$ defined by $f_{k}(x)=I\left(x \in B_{w_{k-1}}\right)-\left(\pi\left(B_{w_{k-1}}\right) / \pi\left(x_{k}\right)\right) I(x=$ $\left.x_{k}\right)$ is an eigenvector for $T$ with eigenvalue $\lambda_{w_{k}}$ for every integer $k \leq$ $N,(k>1$ when $\Omega$ is finite $)$.

(ii) the functions $f_{k}$ together with $\mathbf{1}$ form an orthogonal basis of eigenvectors for $L^{2}(\pi)$, and

(iii) if $\lambda \notin \Lambda \cup\{1\}$, and $\eta=\min \left\{\left|\lambda-\lambda^{\prime}\right|: \lambda^{\prime} \in \Lambda \cup\{1\}\right\}$, then $\|(T-\lambda I) f\| \geq$ $\eta\|f\|$ for any $f \in L^{2}(\pi)$.

Proof: The proof of lemma 2 extends trivially to the countable case, proving (i). Part (ii) follows directly from theorem 1 in the finite case. The orthogonality is trivial also in the countable case. In this case it is easy to see 
that the Dirac function $\delta_{x_{N}}$ is in the space generated by 1 and $f_{N}, \delta_{x_{N-1}}$ is in the space generated by $\mathbf{1}, f_{N}$ and $f_{N-1}$ etc., so that each $\delta_{x_{k}}$ is a finite linear combination of functions $f_{k}$ and 1 . Any $f$ in $L^{2}(\pi)$ can be approximated arbitrarily well by a function of the form $\sum_{k=-K}^{N} f\left(x_{k}\right) \delta_{x_{k}}$, which can be expressed as a linear combination of finitely many $f_{k}$ and $\mathbf{1}$, proving (ii). Hence, clearly there exists an orthonormal basis of eigenvectors for $T$. With respect to this basis, $T-\lambda I$ is represented by a finite or infinite dimensional diagonal matrix with the numbers $\lambda^{\prime}-\lambda$ on the diagonal $\left(\lambda^{\prime} \in \Lambda \cup\{1\}\right)$, proving (iii).

As mentioned in the beginning of section 2, we have to analyse the somewhat artificial case of continuous $\Omega$, but finite or countable $\mathcal{W}$ in order to handle the general continuous case. We restrict the analysis to the case $\mathcal{W}=\left\{w_{k}\right\}$, with $k=1, \ldots, N$ or $k=\ldots,-2,-1,0,1, \ldots, N$, and $w_{k-1}<w_{k}$ if $k<N$, in accordance with the standing assumption of this section

Define for $x \in \Omega$

$$
I(x)=k \text { if } w(x)=w_{k}
$$

Then $\lambda_{w(x)}=\lambda_{w_{I(x)}}$. Recall that we have defined $C_{k}=B_{w_{k}}-B_{w_{k-1}}$. From (3) we can then write

$$
\begin{aligned}
& T f(x)= \sum_{k \leq N} \int_{C_{k}} f(y) q(y) d \mu(y) \\
& \quad+\sum_{k \leq I(x)} \int_{C_{k}}(-q(y)+\pi(y) / w(x)) f(y) d \mu(y)+\lambda_{w(x)} f(x) \\
&= \sum_{k \leq N} q\left(C_{k}\right) \bar{f}(k)+\sum_{k \leq I(x)}\left(-q\left(C_{k}\right)+\pi\left(C_{k}\right) / w_{I(x)}\right) \bar{f}(k)+\lambda_{w_{I(x)}} \bar{f}(I(x)) \\
& \quad+\lambda_{w(x)}(f(x)-\bar{f}(I(x)))
\end{aligned}
$$

where by definition

$$
\bar{f}(k)=\left(1 / q\left(C_{k}\right)\right) \int_{C_{k}} f(y) q(y) d \mu(y)=\left(1 / \pi\left(C_{k}\right)\right) \int_{C_{k}} f(y) \pi(y) d \mu(y),
$$

the last equality following since $\pi$ and $q$ are proportional on $C_{k}$. Let $\Omega_{d}$ be the state space $\{1,2, \ldots, r\}$ or $\{\ldots,-2-1,0,1, \ldots, N\}$, depending on the cardinality of $\mathcal{W}$, and define distributions $q_{d}, \pi_{d}$ on $\Omega_{d}$ by $q_{d}(k)=q\left(C_{k}\right), \pi_{d}(k)=$ 
$\pi\left(C_{k}\right)$. Let $T_{d}$ be the kernel operator for the IMH transition kernel on $\Omega_{d}$ obtained by having $\pi_{d}$ as target distribution and $q_{d}$ as proposal distribution. Note that this gives the same importance weights $w_{k}=\pi_{d}(k) / q_{d}(k)$ as the original IMH. Then (11) can be written in the form

$$
T f(x)=T_{d} \bar{f}(I(x))+\lambda_{w(x)}(f(x)-\bar{f}(I(x)))
$$

Proposition 1 Suppose $\mathcal{W}=\left\{w_{k}\right\}$, where $k=1, \ldots, N$ or $k=\ldots,-2,-1$, $0,1, \ldots, N$, and $w_{k-1}<w_{k}$ for all $k$.

(i) If $f$ is an eigenvector for $T$ with eigenvalue $\lambda$, then either $\lambda=\lambda_{w_{k}}$ for some $k$, and $f$ is 0 on $B_{w_{k}}^{c}$, constant on $B_{w_{k-1}}$ and satisfies

$\int_{C_{k}} f(y) \pi(y) d \mu(y)=-\int_{B_{w_{k-1}}} f(y) \pi(y) d \mu(y)$, where by definition $B_{w_{0}}=\emptyset$ in the finite case, or $\lambda=1, f=1$.

(ii) Every non zero $f$ satisfying the three requirements in (i) is an eigenvector, and every $\lambda_{w_{k}}$ defined by (1) is an eigenvalue, with $\lambda_{w_{1}}=0$ as an exception in the finite case if $w^{-1}\left(w_{1}\right)$ has its entire probability mass concentrated in a single point.

Proof: Suppose that $f$ is an eigenvector with eigenvalue $\lambda$. Then by (13) for every $x$ we have

$$
\begin{aligned}
\lambda f(x) & =\lambda \bar{f}(I(x))+\lambda(f(x)-\bar{f}(I(x))) \\
& =T_{d} \bar{f}(I(x))+\lambda_{w(x)}(f(x)-\bar{f}(I(x)))
\end{aligned}
$$

Integrating both sides of (14) over $C_{k}$ with respect to $q(x) d \mu(x)$ gives $\lambda \bar{f}(k)=$ $T_{d} \bar{f}(k)$. Doing this for each $k$ shows that either $\bar{f}$ is an eigenvector for $T_{d}$ with eigenvalue $\lambda$, or $\bar{f}=0$. Moreover, we get $\lambda(f(x)-\bar{f}(I(x)))=$ $\lambda_{w(x)}(f(x)-\bar{f}(I(x)))$ for every $x$. Hence, either $f$ is constant and equal to $\bar{f}(k)$ on $C_{k}$ for every $k$, or there exists exactly one $k$ such that $f$ may be non constant on $C_{k}$, in which case $\lambda=\lambda_{w_{k}}$. Such a $k$ must exist if $\bar{f}=0$, otherwise $f$ would be identically 0 . In any case, $\lambda=\lambda_{w_{k}}$ for some $k$. We may have $\lambda=\lambda_{w_{1}}=0$ in the case of finite $\mathcal{W}$, in which case $\bar{f}=0$ since, by (i) and (ii) of lemma 3, 0 is not an eigenvalue for $T_{d}$.

It remains to analyse the structure of $f$. Suppose first that $\bar{f}$ is non zero, and hence an eigenvector for $T_{d}$ with eigenvalue $\lambda_{w_{k}}$. Then by lemma $3 \bar{f}(i)=0$ for $i>k, \bar{f}$ takes a constant value $a \neq 0$ for $i<k$, and satisfies 
$\bar{f}(k)=-a \pi\left(B_{w_{k-1}}\right) / \pi\left(C_{k}\right)$. Since for $x \in C_{k}^{c}$ we have $f(x)=\bar{f}(I(x))$, it follows that $f$ is 0 on $B_{w_{k}}^{c}$ and takes the value $a$ on $B_{w_{k-1}}$. Furthermore,

$$
\begin{aligned}
& \int_{C_{k}} f(y) \pi(y) d \mu(y)=\pi\left(C_{k}\right) \bar{f}(k) \\
& =\pi\left(C_{k}\right)\left(-a \pi\left(B_{w_{k-1}}\right) / \pi\left(C_{k}\right)\right)=-a \pi\left(B_{w_{k-1}}\right)=-\int_{B_{w_{k-1}}} f(y) \pi(y) d \mu(y) .
\end{aligned}
$$

Next, suppose that $\bar{f}=0$, then $f(x)=0$ for $x \in C_{k}^{c}$, and $\int_{C_{k}} f(y) \pi(y) d \mu(y)=$ $\pi\left(C_{k}\right) \bar{f}(k)=0=-\int_{B_{w_{k-1}}} f(y) \pi(y) d \mu(y)$. In any case, $f$ and $\lambda$ are as described in (i). Tracing the proof backwards shows that every non zero $f$ as in (i) is an eigenvector, and it is easy to see that there exists a non zero $f$ of this form for every $k$, with the single exception given. This proves (ii).

Let $\|\cdot\|_{d}$ be the norm on the vector space of functions on $\Omega_{d}$ defined by $\pi_{d}$. In order to complete the characterization of the spectrum in the case treated in this section, we need the following "Pythagorean rule":

Lemma 4 For any $f \in L^{2}(\pi)$ we have $\|f\|^{2}=\|\bar{f}\|_{d}^{2}+\|f(\cdot)-\bar{f}(I(\cdot))\|^{2}$.

Proof: We have

$$
\begin{aligned}
\|f\|^{2}= & \int_{\Omega} f(x)^{2} \pi(x) d \mu(x)=\int_{\Omega}[\bar{f}(I(x))+(f(x)-\bar{f}(I(x)))]^{2} \pi(x) d \mu(x) \\
= & \sum_{k \leq N} \bar{f}(k)^{2} \pi_{d}(k)+2 \sum_{k \leq N} \bar{f}(k) \int_{C_{k}}(f(x)-\bar{f}(k)) \pi(x) d \mu(x) \\
& \quad+\int_{\Omega}[f(x)-\bar{f}(I(x))]^{2} \pi(x) d \mu(x) \\
= & \|\bar{f}\|_{d}^{2}+\|f(\cdot)-\bar{f}(I(\cdot))\|^{2}
\end{aligned}
$$

Proposition 2 In the situation of proposition 1

(i) $\Lambda \cup\{1\}$ is the spectrum of $T$.

(ii) If $\lambda \notin \Lambda \cup\{1\}$, then $\|(T-\lambda I) f\| \geq \eta\|f\|$, where $\eta=\min \left\{\left|\lambda-\lambda^{\prime}\right|\right.$ : $\left.\lambda^{\prime} \in \Lambda \cup\{1\}\right\}$.

Proof: Clearly, (i) follows from (ii). To prove (ii), note first that $\overline{T f}=T_{d} \bar{f}$ by (13). The corresponding decomposition of $(T-\lambda I) f$ is therefore

$$
(T-\lambda I) f(\cdot)=\left[\left(T_{d}-\lambda I\right) \bar{f}(I(\cdot))\right]+\left[\left(\lambda_{w(\cdot)}-\lambda\right)(f(\cdot)-\bar{f}(I(\cdot)))\right] .
$$


Hence, using lemma 4, lemma 3, part (iii), and then lemma 4 again, we obtain

$$
\begin{gathered}
\|(T-\lambda I) f\|^{2}=\left\|\left(T_{d}-\lambda I\right) \bar{f}\right\|_{d}^{2}+\left\|\left(\lambda_{w(\cdot)}-\lambda\right)(f(\cdot)-\bar{f}(I(\cdot)))\right\|^{2} \\
\geq \eta^{2}\|\bar{f}\|_{d}^{2}+\eta^{2}\|f(\cdot)-\bar{f}(I(\cdot))\|^{2}=\eta^{2}\|f\|^{2} .
\end{gathered}
$$

\section{The general continuous case}

In this section we assume that $\Omega$ is an arbitrary continuous state space. The key to solving this case is to approximate $q$ with proposal densities $q^{n}$ for which the results of the previous section can be applied. Details are given in the following quite technical lemma:

Lemma 5 Let $q$ be a proposal density with corresponding kernel operator $T$. Then there exists a sequence $\left(q^{n}\right)$ of proposal densities and corresponding sequences of

(i) sets $\mathcal{W}^{n}=\left\{w_{k}^{n}, k \in \Omega_{d}^{n}\right\}$, where $\Omega_{d}^{n}$ is either finite, of the form $\left\{1,2, \ldots, N^{n}\right\}$, or countable, of the form $\left\{\ldots,-2,-1,0,1, \ldots, N^{n}\right\}$,

(ii) functions $I^{n}: \Omega \rightarrow \Omega_{d}^{n}$,

(iii) kernel operators $T^{n}$

(iv) sets of eigenvalues $\lambda_{w_{k}^{n}}^{n}, k \in \Omega_{d}^{n}$ for $T^{n}$, such that

(i) $q^{n}(x)=\pi(x) / w_{I^{n}(x)}^{n}$

(ii) $\left\|T^{n}-T\right\| \rightarrow 0$ as $n \rightarrow \infty$

(iii) $\sup _{x}\left(\left|\lambda_{w_{I^{n}(x)}^{n}}^{n}-\lambda_{w(x)}\right| \rightarrow 0\right.$ as $n \rightarrow \infty$.

Proof: Assume first that $0<w_{*}<w^{*}<\infty$. For each $n$ we define numbers $0<v_{0}^{n}<w_{*}=v_{1}^{n}, \ldots, v_{L^{n}}^{n}=w^{*}$ inductively by putting $v_{l+1}^{n}=\min \left\{v_{l}^{n}+\right.$ $\left.\left(v_{l}^{n}\right)^{2} / n, w^{*}\right\}$ if $v_{l}^{n}<w^{*},(l=1,2, \ldots)$. We also define inductively disjoint subsets $C_{k}^{n}, k=1, \ldots, N^{n}$ as follows: Set $C_{1}^{n}=B_{v_{l}^{n}}-B_{v_{l-1}^{n}}$ for the smallest $l \geq 1$ such that $\pi\left(B_{v_{l}^{n}}-B_{v_{l-1}^{n}}\right)>0$. Assume $C_{k}^{n}=B_{v_{s}^{n}}-B_{v_{s-1}^{n}}$ has been defined. Put $t=\min \left\{l>s: \pi\left(B_{v_{l}^{n}}-B_{v_{l-1}^{n}}\right)>0\right.$, and define $C_{k+1}^{n}=$ 
$B_{v_{t}^{n}}-B_{v_{t-1}^{n}}$. Let $w_{k}^{n}=\pi\left(C_{k}^{n}\right) / q\left(C_{k}^{n}\right)$, and define $q^{n}(x)=\pi(x) / w_{k}^{n}$ for $x \in C_{k}^{n}$, which by definition means that $I^{n}(x)=k$. Clearly, $w^{-1}(\mathcal{W}) \subseteq \cup_{k=1}^{N^{n}} C_{k}^{n}$, so that by our standard assumption that $w^{-1}(\mathcal{W})=\Omega$ it follows that $q^{n}$ and $I^{n}$ are defined on all of $\Omega$. Clearly, $q^{n}\left(C_{k}^{n}\right)=q\left(C_{k}^{n}\right)$ for each $k$, and it follows that $q^{n}$ defines a probability distribution. For this distrubution the set $\mathcal{W}^{n}=\left\{\pi(x) / q^{n}(x): x \in \Omega\right\}=\left\{w_{1}^{n}, \ldots, w_{N^{n}}^{n}\right\}$ is finite. Let $T^{n}$ be the corresponding kernel operator.

Fix $x \in C_{k}^{n}=B_{v_{l}^{n}}-B_{v_{l-1}^{n}}$. We then have $v_{l-1}^{n}<w(x) \leq v_{l}^{n}$, and $v_{l-1}^{n} \leq w_{k}^{n} \leq v_{l}^{n}$. It follows that

$$
\left|1 / w(x)-1 / w_{k}^{n}\right|=\left|w(x)-w_{k}^{n}\right| / w(x) w_{k}^{n} \leq\left(v_{l}^{n}-v_{l-1}^{n}\right) /\left(v_{l-1}^{n}\right)^{2} \leq 1 / n
$$

and also

$$
\left|q(x)-q^{n}(x)\right|=\left|\pi(x) / w(x)-\pi(x) / w_{k}^{n}\right| \leq \pi(x) / n
$$

Put $B_{k}^{n}=\cup_{j=1}^{k} C_{k}^{n}$. Then $B_{k-1}^{n}=B_{k}^{n}-C_{k}^{n} \subseteq B_{w(x)} \subseteq B_{k}^{n}$. Hence, the eigenvalue $\lambda_{w_{k}^{n}}^{n}$ for $T^{n}$ (see (ii) of proposition 1) is given by

$$
\lambda_{w_{k}^{n}}^{n}=\int_{B_{k}^{n}}\left(q^{n}(y)-\pi(y) / w_{k}^{n}\right) d \mu(y)=\int_{B_{w(x)}}\left(q^{n}(y)-\pi(y) / w_{k}^{n}\right) d \mu(y) .
$$

It follows that for any $x$ we have

$$
\begin{aligned}
& \left|\lambda_{w(x)}-\lambda_{w_{I^{n}(x)}^{n}}^{n}\right|=\left|\int_{B_{w(x)}}\left[(q(y)-\pi(y) / w(x))-\left(q^{n}(y)-\pi(y) / w_{I^{n}(x)}^{n}\right)\right] d \mu(y)\right| \\
& \leq \int_{B_{w(x)}}\left[\left|q(y)-q^{n}(y)\right|+\mid \pi\left(y / w(x)-\pi(y) / w_{I^{n}(x)}^{n} \mid\right] d \mu(y) \leq 2 / n\right.
\end{aligned}
$$

where the last inequality follows from (15) and (16). Hence, $\lambda_{w_{I^{n}(\cdot)}^{n}}^{n}$ converges uniformly to $\lambda_{w(\cdot)}$, as asserted.

To prove that $T^{n} \rightarrow T$, choose an arbitrary $f \in L^{2}(\pi)$. Then

$$
\begin{aligned}
T f(x)- & T^{n} f(x)=\int_{\Omega}\left(q(y)-q^{n}(y)\right) f(y) d \mu(y) \\
+ & \int_{B_{w(x)}}\left(-q(y)+\pi(y) / w(x)+q^{n}(y)-\pi(y) / w_{I^{n}(x)}^{n}\right) f(y) d \mu(y) \\
& +\left(\lambda_{w(x)}-\lambda_{w_{I^{n}(x)}^{n}}^{n}\right) f(x) .
\end{aligned}
$$


It follows by (15), (16) and (17) that

$$
\begin{gathered}
\left|T f(x)-T^{n} f(x)\right| \leq \int_{B_{w(x)}^{c}} \mid\left(q(y)-q^{n}(y) f(y)\left|d \mu(y)+\int_{B_{w(x)}}\right|(\pi(y) / w(x)\right. \\
\left.\quad-\pi(y) / w_{I^{n}(x)}^{n}\right) f(y)|d \mu(y)+(2 / n)| f(x) \mid \\
\leq(1 / n)\left(E_{\pi}(|f(Y)|)+2|f(x)|\right)
\end{gathered}
$$

Consequently, using the Cauchy - Schwarz inequality we obtain

$$
\begin{aligned}
\left\|T f-T^{n} f\right\|^{2} & \leq(1 / n)^{2} \int_{\Omega}\left(E_{\pi}(|f(Y)|)+2|f(x)|\right)^{2} \pi(x) d \mu(x) \\
& =(1 / n)^{2}\left(5 E_{\pi}(|f(Y)|)^{2}+4\|f\|^{2}\right) \leq\left(9 / n^{2}\right)\|f\|^{2} .
\end{aligned}
$$

This completes the proof if $0<w_{*}<w^{*}<\infty$. If $w^{*}=\infty$, the finite sequence $v_{l}^{n}, l=1,2, \ldots, L^{n}$ is terminated by requiring $v_{L^{n}}^{n} \geq n$. The corresponding sequence $w_{k}^{n}, k=1,2, \ldots, N^{n}$ is defined as before, except that the largest value is given by $w_{N^{n}}^{n}=\pi\left(B_{v_{L^{n}}^{n}}^{c}\right) / q\left(B_{v_{L^{n}}^{n}}^{c}\right)$. For $x \in C_{N^{n}}^{n} \stackrel{\text { def }}{=} B_{v_{L^{n}}^{n}}^{c}$ we have

$$
\begin{aligned}
\left|1 / w(x)-1 / w_{N^{n}}^{n}\right| & \leq \max \left\{w(x), w_{N^{n}}^{n}\right\} / w(x) w_{N^{n}}^{n} \\
& =1 / \min \left\{w(x), w_{N^{n}}^{n}\right\} \leq 1 / v_{L^{n}}^{n} \leq 1 / n
\end{aligned}
$$

Hence, the inequality (15) is satisfied for every $x$ also in this case, and the proof proceeds as before.

Finally, assume $w_{*}=0$. We then construct a sequence $v_{l}^{n}, l=\ldots,-2,-1$, $0,1, \ldots, L^{n}$ in the following way: Set $v_{L^{n}}^{n}=\min \left\{n, w^{*}\right\}$. Proceed by backward induction to find $v_{l}^{n}$ such that $v_{l+1}^{n}=v_{l}^{n}+\left(v_{l}^{n}\right)^{2} / n, l=L^{n}-1, L^{n}-2, \ldots$. This amounts to solving a simple quadratic equation, yielding $v_{l}^{n}=\left(n v_{l+1}^{n}+\right.$ $\left.n^{2} / 4\right)^{1 / 2}-n / 2$. The sequence $v_{l}^{n}$ decreases as $l$ decreases. If there existed a lower bound $v>0$, the difference between consecutive elements would converge to $v^{2} / n>0$, a contradiction. Hence, $v_{l}^{n} \rightarrow 0$ as $l \rightarrow-\infty$. Arranging those sets among the sets $B_{v_{l+1}}-B_{v_{l}}$ that are non empty in a sequence $C_{k}^{n}, k=\ldots,-2,-1,0,1, \ldots, N^{n}$ as before, we therefore have that $\cup_{k \leq N^{n}} C_{k}^{n}=\Omega$ (the integers $L^{n}, N^{n}$ can be chosen arbitrarily in this case). This is all that is needed in order to develop the proof completely in parallel to the case already treated.

Theorem 2 The spectrum of $T$ equals $\Lambda \cup\{1\}=\left\{\lambda_{w}: w \in \mathcal{W}\right\} \cup\{1\}$. 
Proof: Suppose $\lambda=\lambda_{w} \in \Lambda$. Choose a sequence $\left(x_{n}\right)$ such that $w\left(x_{n}\right) \rightarrow w$. Then also $\lambda_{w\left(x_{n}\right)} \rightarrow \lambda_{w}$. Construct a sequence of operators $T^{n}$ and sets of eigenvalues $\lambda_{w_{k}^{n}}^{n}$ as in lemma 5. Then $\lambda_{w_{I^{n}\left(x_{n}\right)}^{n}}^{n} \rightarrow \lambda_{w}=\lambda$. It follows that the sequence $T^{n}-\lambda_{w_{I^{n}\left(x_{n}\right)}^{n}}^{n} I$ of non invertible operators converges to $T-\lambda I$, which is consequently also non invertible.

Now suppose $\lambda \notin \Lambda, \lambda \neq 1$. Since $\Lambda$ is closed, or at least intersects with $[0, a]$ in a closed set for every $a<1$, it follows that $\lambda$ has a positive distance $\eta>0$ to $\Lambda$. Constructing sequences of operators $T^{n}$ and sets of eigenvalues $\lambda_{k}^{n}$ by lemma 5 again, we have $\left\|T^{n}-T\right\|<\eta / 4$ and $\left|\lambda_{k}^{n}-\lambda\right|>3 \eta / 4$ for every $k$ if $n$ is sufficiently large. For any $f$ with $\|f\|>0$ we then have by proposition 2, part (ii)

$$
\|(T-\lambda I) f\| \geq\left\|\left(T^{n}-\lambda I\right) f\right\|-\left\|\left(T^{n}-T\right) f\right\|>(3 \eta / 4-\eta / 4\|f\|=(\eta / 2)\|f\| .
$$

Consequently $T-\lambda I$ is invertible, and $\lambda$ is not in the spectrum of $T$.

A close examination of the proofs shows that theorem 2 is valid also in mixed continuous/discrete cases, except in the very special case when $w_{*}>0$ is an isolated point in $\mathcal{W}$ and there exists a state $x$ with $\pi(x)>0$ such that $w(x)=w_{*}$, and $\pi\left(w^{-1}\left(w_{*}\right)\right)=\pi(x)$. Then $\lambda_{w_{*}} \in \Lambda$ is not in the spectrum, just as in the finite case (see (ii) of proposition 1 ).

\section{Acknowledgements}

I am very greatful to professor Bent Natvig for his careful reading of the manuscript and many suggestions and comments. Useful comments were also provided by professors Inge Helland and Arnoldo Frigessi. I would also like to thank professor Nils Henrik Risebro for helpful discussions.

\section{References}

GÅSEMYR, J. (2004). A theoretical analysis of the performance of the independent Metropolis-Hastings algorithm on continuous state spaces. (In progress).

HAUGE, R. (2002). On convergence of independent Metropolis- Hastings. Norwegian Computing Center, P.O.Box 114 Blindern, N-0314 Oslo, Norway. HOLDEN, L, HAUGE, R. and HOLDEN, M. (2002). Adaptive independent Metropolis-Hastings. Norwegian Computing Center, P.O.Box 114 Blindern, N-0314 Oslo, Norway. 
HASTINGS, W. K. (1970). Monte Carlo sampling methods using Markov chains and their applications. Biometrica 57, 97 - 109.

LIU, J. S. (1996). Metropolized independent sampling with comparison to rejection sampling and importance sampling. Statist. Comput. 6, 113 - 119. METROPOLIS, M., ROSENBLUTH, A. W., ROSENBLUTH, M. N., TELLER, A. and TELLER, E. (1953). Equation of state calculations by fast computing machines. J. Chem. Fys. 21, 1087 - 1092.

TIERNEY, L. (1994). Markov chains for exploring posterior distributions. Ann. Statistics 22, 1701 - 1762. 